\title{
Perinatally Acquired Tuberculosis in a Neonate
}

\section{A Case Report}

\author{
M.S. Hammoud a,b S.O. Maiyeguna F.M. Al Saban ${ }^{a}$ L.V. Devarajan ${ }^{a, b}$ \\ A.H. Malik ${ }^{a}$ E.M. Hanafic \\ a Neonatal Department, Al Sabah Maternity Hospital, bDepartment of Pediatrics, Faculty of Medicine, \\ Kuwait University, and ' Department of Pediatrics, Al-Adan Hospital, Kuwait
}

\section{Key Words}

Tuberculosis, perinatal $\cdot$ Kuwait

\begin{abstract}
Objectives: To report a case of perinatal tuberculosis that appeared on the 21th day of life of an infant born to a mother with latent tuberculosis. Clinical Presentation and Intervention: A preterm male infant was born by spontaneous vertex delivery at 33 weeks gestational age to a 33-year-old primiparous Philippine woman. The infant was well until the 21 st day of life when he developed recurrent episodes of cyanosis and bradycardia. A chest radiograph showed infiltrates which were thought to be bacterial in origin. Blood, urine, and cerebrospinal fluid cultures were normal. Tracheal aspirate revealed acid-fast bacilli by Ziehl-Neelsen stain, later confirmed to be Mycobacterium tuberculosis by culture in Lowenstein-Jensen medium. The mother was later diagnosed as a case of tuberculosis with symptoms, signs and radiologic manifestation of hilar lymphadenopathy with mild pleural effusion and positive tuberculin skin test. Both infant and mother were treated with intravenous isoniazid, intravenous rifampicin, oral pyrazinamide, and intravenous pyridoxine. Both recovered. Conclusion: A
\end{abstract}

\section{KARGER}

Fax +41613061234

E-Mail karger@karger.ch

www. karger.com
(C) 2004 S. Karger AG, Basel

1011-7571/04/0132-0107\$21.00/0

Accessible online at:

www. karger.com/mpp preterm male infant perinatally acquired tuberculosis, most likely by inhalation of the bacteria during delivery. Both infant and mother responded well to antituberculous treatment.

Copyright $\odot 2004$ S. Karger AG, Basel

\section{Introduction}

Since the early 1900 s, the term congenital tuberculosis has been used to refer to tuberculosis in infants that arises from an intrauterine source of infection. The transmission may occur in utero (congenital transmission) or during delivery (perinatal transmission). It is a rare disease with a high mortality rate of approximately $50 \%[1,2]$; birth is usually premature $[1,3]$, although term deliveries have been described $[4,5]$. Perinatal transmission can be by aspiration, ingestion of infected amniotic fluid, hematogenous spread, endometritis [6], or rarely through ingestion of breast milk. Fatal cases with systemic dissemination usually present early, during the first 2-3 weeks of life [6-9]. Before 1950 the condition was always fatal, but since the introduction of isoniazid (INH) in 1960, there has been an increase in the number of reports of children successfully treated for tuberculosis.
Dr. Majeda Sabri Hammoud

Department of Pediatrics, Faculty of Medicine

Kuwait University, PO Box 24923

13110 Safat (Kuwait)

Tel./Fax +965 5338940, E-Mail m.hammoud@hsc.kuniv.edu.kw 
Fig. 1. Bronchopneumonic changes due to M. tuberculosis.

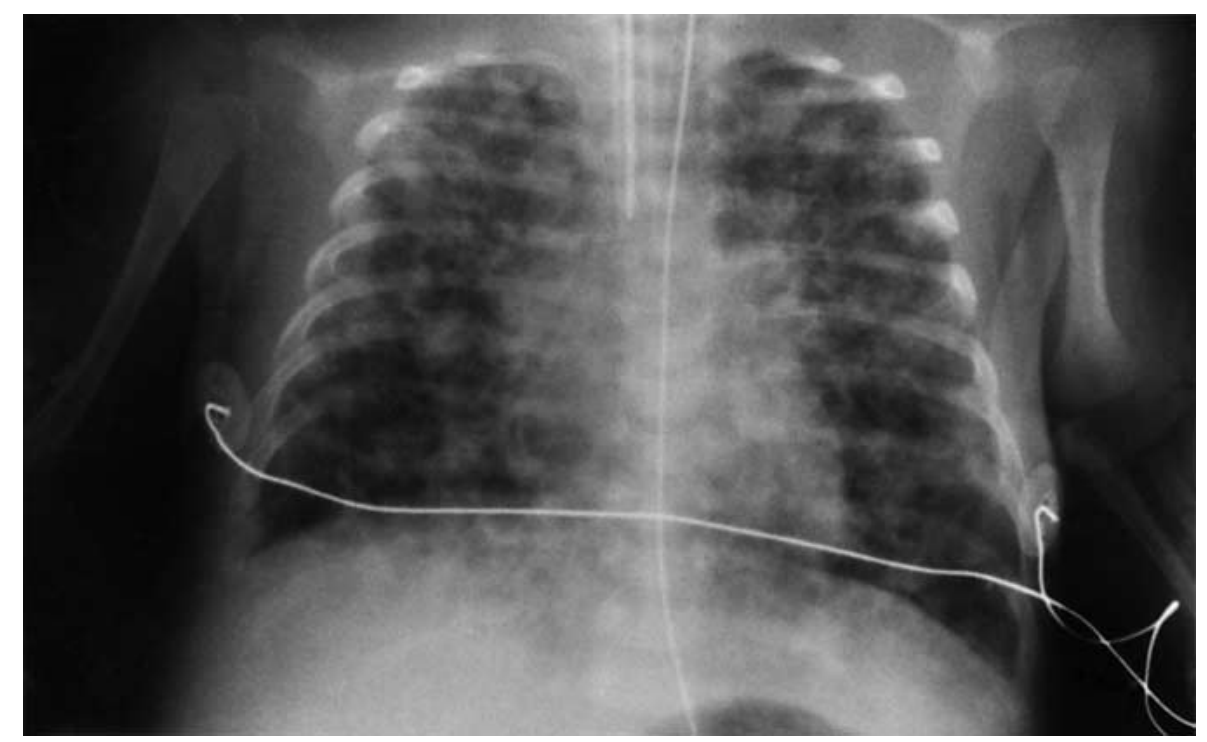

The exact incidence of congenital tuberculosis is unknown due to the difficulty in accurate and early diagnosis. During the past two decades, isolated cases of tuberculosis in neonates have been reported from various parts of the world $[7,8]$. We report such a case from Kuwait, an Arabian Gulf country.

\section{Case Report}

A preterm, male infant was born by spontaneous vertex delivery at 33 weeks gestation to a 33-year-old, primiparous Philippine woman. The pregnancy and delivery were uneventful. The infant was well at birth with Apgar scores of 8 and 9 at 1 and 5 min, respectively. He was normal for the gestational age of 33 weeks; birth weight: $1,535 \mathrm{~g}$; head circumference: $30 \mathrm{~cm}$; length: $42 \mathrm{~cm}$. He was nursed in an incubator and made satisfactory progress initially with no respiratory problems. Nasogastric feeding began with preterm formula, as breast milk was not available.

The infant thrived well until the 21 st day of life when he developed recurrent episodes of cyanosis and bradycardia. Blood, urine and cerebrospinal fluid (CSF) cultures were normal. A chest radiograph showed infiltrates thought to be bacterial in origin (fig. 1). Initial liver function test revealed alanine amino transferase of $85 \mathrm{U} / \mathrm{l}$, alkaline phosphatase $200 \mathrm{U} / \mathrm{l}$, conjugated bilirubin of more than $20 \%$ of the total bilirubin, and albumin $35 \mathrm{~g} / 1$. Complete white blood cell count initially was $6 \times 10^{9} / 1$ and later increased to $30 \times 10^{9} / 1$. The white cells were less than 5 neutrophils, and protein was less than $2 \mathrm{~g} / \mathrm{dl}$. Erythrocyte sedimentation rate was within acceptable limits $(8 \mathrm{~mm} / \mathrm{h})$. The CSF showed no acid-fast bacilli (AFB) by Ziehl-Neelsen stain, and CSF glucose was greater than $50 \%$ of the concurrent blood glucose. On the 21 st day of life, the infant was started on ampicillin at $100 \mathrm{mg} / \mathrm{kg} /$ day given every $12 \mathrm{~h}$ and gentamycin at $2.5 \mathrm{mg} /$ $\mathrm{kg} /$ dose every $12 \mathrm{~h}$. As there was no improvement, on the $32 \mathrm{nd}$ day the antibiotic therapy was stopped and the infant was started empirically on amphotericin B.

Repeat chest radiograph showed diffuse bilateral nodular opacifications. With a high index of suspicion of tuberculosis infection, the infant was intubated; both tracheal and gastric aspirates were stained for AFB by Ziehl-Neelsen stain and culture. The tracheal aspirate revealed numerous AFB, later confirmed to be Mycobacterium tuberculosis by culture in Lowenstein-Jensen (L-J) medium. A Mantoux test was performed with $0.1 \mathrm{ml}$ of 5 tuberculin units of purified protein derivative given intradermally into the volar aspect of the forearm, and the skin reaction after $72 \mathrm{~h}$ was negative. Further CSF collected 3 times at weekly intervals showed no AFB on staining and culture. His condition suddenly deteriorated and required assisted ventilation, which lasted for 3 weeks.

On the 36th day of life he was started on intravenous rifampicin at $10 \mathrm{mg} / \mathrm{kg} / \mathrm{day}$, intravenous INH at $10 \mathrm{mg} / \mathrm{kg} /$ day, oral pyrazinamide at $20 \mathrm{mg} / \mathrm{kg} / \mathrm{day}$, and intravenous pyridoxine at $50 \mathrm{mg}$ daily. Hepatosplenomegaly first noticed at 3 weeks of life regressed gradually to normal size on follow-up. The chest radiographic improvement lagged behind the clinical response. He continued to thrive on oral foods, gained weight and was extubated at the age of 53 days. Pyrazinamide was given for 2 months and the infant was discharged at 3 months of age on INH and rifampicin to complete 12 months of treatment at home. The mother gave the medication at home, although the World Health Organization (WHO) and Centers for Disease Control and Prevention recommend the use of the directly observed therapy, whereby treatment is provided directly to the patient by a health care worker on a trained third party (not a relative or a friend) to document that the patient takes each dose of medication. Directly observed therapy is not a common practice in Kuwait. The infant was seen with the mother monthly and chest X-ray was done every 3 months.

As for the mother, she had a history of recurrent fever, chills and night sweats since the delivery of her baby. She was diagnosed with tuberculosis with symptoms, signs and radiologic manifestation of hilar lymphadenopathy with mild pleural effusion, and a tuberculin 
skin test of more than $15 \mathrm{~mm}$ induration. Her sputum and early morning gastric lavage were positive for AFB and M. tuberculosis on L- J culture. AFB stain and L-J medium culture of endometrial curettage were not positive for $M$. tuberculosis. The placenta was not examined at delivery, because there was no suspicion of tuberculosis in the mother during antenatal care. Human immunodeficiency virus (HIV) status of both mother and baby was negative.

The mother was treated with an oral three-drug regimen of INH at $300 \mathrm{mg}$ daily, pyrazinamide at $2.0 \mathrm{~g}$ daily and rifampicin at $600 \mathrm{mg}$ daily. Two months after tuberculosis treatment began, her sputum became negative to AFB, and she was discharged on INH and rifampicin to complete 6 months of antituberculous therapy. The drugs were self-administered. One month after discharge from the hospital, both mother and infant were in good general condition and continued on antituberculous treatment.

The community health department carried out tuberculin skin test (TST) on 8 health workers at the neonatal intensive care unit and 30 workers at the special care baby unit, as well as close family members who were present during the period including the father, maid and mother's sister. All of the TSTs, which were repeated after 3 months, proved negative. Of the 20 infants in the hospital units who fulfilled the criteria for exposure [being present in the same room with the mother (index case) cumulatively for $24 \mathrm{~h}$ ], 12 infants were still in the unit when the diagnosis was made and 8 had been discharged from the neonatal department. The exposed infants had TST and chest radiographs and were further screened with gastric aspirates; all were negative and as such none received INH treatment based on the recommendation of our community health department.

\section{Discussion}

Perinatal tuberculosis is rare and is generally acquired during birth by inhalation of infected material or immediately after birth through attendants such as infected midwife, parent or other adults. In this case, all of the health care workers and close family members, including the father, maid and mother's sister, had negative TSTs, while the mother tested positive and was therefore the suspected source of infection in the neonate. The infection could have been transmitted to the fetus by hematogenous spread from the placenta to the umbilical vein or by aspiration or ingestion of amniotic fluid contaminated by placental or genital infection [5]. Since tuberculosis was diagnosed in the mother after the baby was born, it is likely that the bacterium was transmitted during delivery and not in utero.

As further evidence that the tuberculosis was acquired perinatally and not congenitally, the baby did not meet the diagnostic criteria for congenital tuberculosis as described by Cantwell et al. [5]. These criteria require that the infant have at least one of the following: lesions in the 1st week of life; a primary hepatic complex or caseating hepatic granuloma; tuberculous infection of the placenta or the maternal genital tract; or exclusion of the possibility of postnatal transmission by thorough investigation of contacts including the infant's hospital attendants. These criteria were not applicable in this case, but it should be noted that the placenta was not checked since the mother did not show any signs or symptoms of tuberculosis during pregnancy. It can be argued that the infection was latent and not transferred across the placenta, since the mother did not have the disease during pregnancy and the baby was not sick at birth.

Therefore in this case of severe pulmonary tuberculosis and probably miliary spread to other organs like the liver, the infant most likely inhaled the bacteria during delivery, which caused a tuberculous bronchopneumonia followed by dissemination which appeared after 3 weeks. The patient's TST was negative in spite of the severe infection, which is expected in infants with tuberculosis [10], especially in a premature infant. Regardless of how the tuberculosis was acquired, the treatment is the same.

Tuberculosis should be suspected in the infant when the mother has a history of tuberculosis, risk factors for the disease, unexplained fever, pleurisy or night sweats. Also, it should be suspected if the infant has pneumonia unresponsive to the usual antibiotics $[8,9]$. Proof of infection in the newborn can be established by finding AFB in body fluids, tissue or by culturing $M$. tuberculosis. A positive AFB from early morning gastric aspirate in a newborn indicates tuberculosis [7-9]. AFB from tracheal aspirate, bone marrow, or biopsy tissue can be vital in the diagnosis. Mantoux tuberculin skin test is not reliable in infants less than 6 months of age, and it is even less reliable in neonates because of their immature immune system [10-12], as evidenced in this case.

A treatment regimen of four drugs including INH, rifampicin, pyrazinamide and ethambutol or streptomycin in areas where resistant tuberculosis is above $4 \%$ is recommended [13, 14]. Since tuberculosis is under strict control in Kuwait because of regular screening of all expatriates and drug resistance was not a concern, streptomycin was not added to the initial three-drug regimen in this case. Although the mother is from the Philippines where resistant tuberculosis is highly prevalent, she possibly had a reactivation of a latent infection after delivery of the child.

INH is the basis for therapy and should be continued for 9-12 months. Steroid therapy is not usually indicated, as the host reaction is minimal [7]. INH prophylaxis is often indicated for healthy infants born to mothers with active tuberculosis and for those who have been in recent contact with a person who has active tuberculosis, when 
clinical disease has been excluded, even if TST result is negative especially in infants, young children and those with HIV [12-14]. Prophylactic protocols differ throughout the world $[10,15]$. In countries with high prevalence of tuberculosis, Bacillus Calmette-Guérin vaccination is given at or within days of birth. It has some protective effect against the development of tuberculosis in the newborn and appears to decrease the incidence of life-threatening and invasive forms of the disease [15].

Once perinatal tuberculosis is diagnosed, the risk of spread of tuberculosis via the respiratory route to the health care workers and other infants is very low, as the infants have no forceful cough to disseminate the organism [12].

\section{Conclusion}

A preterm male infant perinatally acquired tuberculosis, most likely by inhalation of the bacteria during delivery. Both infant and mother responded well to antituberculous treatment.

\section{References}

1 Pham Duy L, Le Van N, Truong Ngoc Cuc H: Congenital miliary tuberculosis: A propos of a case. Rev Pneumol Clin 1998;54:207-209.

2 Aireda KI: Congenital miliary tuberculosis. Ann Trop Paediatr 1990;10:363-368.

3 Pillet P, Grill J, Rakotonirina G, Holvoet-Vermaut L, Auregan G, Guyon P: Congenital tuberculosis: Difficulties in early diagnosis. Arch Pediatr 1999;6:635-639.

4 Hageman J, Shulman S, Schreiber M, Luck S, Yogev R: Congenital tuberculosis: Critical reappraisal of clinical findings and diagnostic procedures. Pediatrics 1980;66:980-984.

5 Cantwell MF, Shehab ZM, Costello AM, Sands L, Green WF, Ewing EP Jr, Valway SE, Onorato IM: Brief report: Congenital tuberculosis. N Engl J Med 1994;330:1051-1054.

6 Beitzke H: Über die angeborene Tuberkuloseinfektion. Ergeb Tuberk Forsch 1935;13:343353.
7 Abughali N, Van der Kuyp F, Annable W, Kumar ML: Congenital tuberculosis. Pediatr Infect Dis J 1994; 13:738-741.

8 Machin GA, Honore LH, Fanning EA, Molesky MM: Perinatally acquired neonatal tuberculosis: Report of two cases. Pediatr Pathol 1992; 12:707-716.

9 Smith KC: Congenital tuberculosis: A rare manifestation of a common infection. Curr Opin Infect Dis 2002;15:269-274.

10 Starke JR: Childhood tuberculosis: Treatment strategies and recent advances. Pediatr Respir Rev 2001;2:103-112.

11 Smith KC, Starke JR, Eisenbach K, Ong LT, Denby M: Detection of Mycobacterium tuberculosis in clinical specimen from children using a polymerase chain reaction. Pediatrics 1996; 97:155-160.
12 Lee LH, leVea CM, Graman PS: Congenital tuberculosis in a neonatal intensive care unit: Case report, epidemiological investigation, and management of exposures. Clin Infect Dis 1998;27:474-477.

13 American Academy of Pediatrics (Tuberculosis): In Pickering LK (ed): 2000 Red Book: Report of the Committee on Infectious Disease, ed 25. Elk Grove Village, American Academy of Pediatrics, 2000, pp 593-618.

14 Maher D, Chaulet P, Spinaci S, Harries A: Treatment of Tuberculosis: Guidelines for National Programmes, ed 2. Bull WHO, 1997, pp 41-48.

15 Colditz G, Berkey C, Mosteller F, et al: The efficacy of Bacillus Calmette-Guérin vaccination of newborn and infants in the prevention of tuberculosis: Meta-analyses of the published literature. Pediatrics 1995;96:29-35. 